\title{
Perceived causes of stress among a group of western Canadian dental students
}

\author{
Alyssa Hayes ${ }^{1 *} \mathbb{D}$, Jay N. Hoover ${ }^{1}$, Chandima P. Karunanayake² and Gerald S. Uswak
}

\begin{abstract}
Objective: The demanding nature of dental education, both academically and clinically, results in higher levels of perceived stress among its students. The aim of this study was to determine how dental students at the College of Dentistry, University of Saskatchewan perceived stress. During the 2013-2014 academic year, all students were asked to complete a modified dental environmental survey (DES).

Results: Of the 111 students enrolled at the College that year 92 completed the survey (response rate $=83 \%$ ). In general, female students reported higher stress levels than males. Higher stress levels were associated with living away from home, concerns about manual dexterity and the transition from pre-clinical to clinical studies. Additionally, students who enter dental school with higher debt loads $(>100,000)$ report high stress levels relating to finances. This study found that financial and clinical workloads result in high stress levels among dental students.
\end{abstract}

Keywords: Stress, Dental education, Dental students

\section{Introduction}

The Canadian Mental Health Association (CMHA) defines stress as "the body's response to a real or perceived threat [...] [however], most of the threats people face today $[\ldots]$ are usually problems that people have to work through" [1]. They go on to state that stress can be both helpful (in terms of motivating people towards problem or task completion) or unhelpful, which is some instances can be associated with problem or task avoidance and physical symptoms (e.g., increased heart rate, sweating, headaches and sleeping difficulties) [1]. Often associated with major life events (i.e., entering a professional school, changing jobs) responses reflect a person's perception about their ability to handle the situation. Dental education, like other health professional programs, is considered to be demanding both, academically and clinically and is associated with higher levels of stress among students [2]. The literature has shown that stress among dental students is associated with emotional exhaustion, burnout, decreased productivity and lower

\footnotetext{
*Correspondence: alyssa.hayes@usask.ca

${ }^{1}$ College of Dentistry, University of Saskatchewan, 105 Wiggins Rd,

Saskatoon, SK S7N 5E4, Canada

Full list of author information is available at the end of the article
}

academic success (lower GPA) [2-4]. Common stressors include marital status, gender, academic year, personality type, clinical training and financial burden [5-7]. Al-Saleh et al. [7] reported that among Saudi dental students, stress levels peaked during the latter years of dental training and were associated with the availability of patients, treatment compliance and supervisor feedback. While there are common stressors (i.e., clinical training, patient treatment and supervisor feedback) identified in the literature, prevalence and impact on student stress levels varies and appears to be related to the teaching institution [6]. In light of these variations and the scarcity of Canadian data on this topic, this study attempted to ascertain sources of perceived stress in undergraduate dental students at the University of Saskatchewan, College of Dentistry.

\section{Main text}

\section{Materials and methods}

All undergraduate dental students enrolled in the Doctor of Dental Medicine (DMD) program at the College of Dentistry, University of Saskatchewan, located in western Canada, were invited to participate in the study. Data collection occurred between January and February 2014, thus allowing for the completion of one semester. 
This study received ethical approval from the Behaviorial Research Ethics Board (REB), University of Saskatchewan (BEH \#12-122). Written informed consent was obtained prior to data collection. Paper-based surveys (see Additional file 1) were administered to all years by a College staff member to ensure anonymity. A modified version of the dental environmental stress (DES) questionnaire, and a 10-item Perceived Stress Scale questionnaire (PSS-10) were used [8]. Both instruments are widely accepted, and have proven to be effective tools to quantify stress levels. Data were collected regarding demographics and motivation for choosing a dental career, analysis was completed using SPSS version 24 (SPSS Inc., Armonk, NY: IBM Corp.). Descriptive statistics were used to describe the study and outcome variables. Internal consistency of the questionnaire was assessed by calculating Cronbach's alpha. A Kruskal-Wallis test [9] was employed to determine significant differences between year of study, gender, marital status, grade point average, ethnicity, and debt load and debt interest payment. The level of significance was set at $\mathrm{p}<0.05$.

\section{Results}

The reliability of the questionnaire with all items (49) was 0.959, which indicated significant internal consistency (see Additional file 2). Scale 1 contained 10 items referring to social-related stress (Cronbach's alpha for internal consistency between the items was 0.802); scale 2 (7 items) dealt with financial related stress (Cronbach's alpha $=0.902)$; scale 3 (11 items) related to clinicalrelated stress (Cronbach's alpha $=0.851$ ); and the fourth scale (21 items) represented academic-related stress (Cronbach's alpha $=0.934$ ). The adequacy of items under each of the factors was also assessed by calculating the range of Cronbach's alpha values.

At the time the study was completed, the College of Dentistry had 111 students enrolled in all years, with a response rate of 83 percent the survey is representative of the student body. The dental student cohort at the College of Dentistry at the time of the study was mostly male (56.5\%). Respondents were representative of each academic year of the dental program (see Additional file 3). The majority of respondents were between 23 and 25 years of age $(55.4 \%)$ and reported being never married or single $(66.3 \%)$. Of interest is that almost 58 percent reported having a current debt load of greater than $\$ 100,000(\mathrm{CDN})$.

Table 1 shows that when comparing between each academic years statistical differences in stress levels were seen for: socially related stressors (living away from home); clinical related stressors (concern about manual dexterity and clinical skills, the transition from pre- clinical to clinical studies); and academic related stressors (communication with faculty or staff, fear of failing a course/year, confidence about own decision making, and student's input into College decision-making) were significantly different $(\mathrm{p}<0.05)$. Among first year dental students, the highest mean DES score was attributed to fear over failing a course or year (mean $\mathrm{DES}=4.36$; SD 1.07). This level of fear decreased as students progressed through their education, with fourth year students reporting considerably lower levels (mean $\mathrm{DES}=2.16$, SD 1.71). Additionally, social-related stressors (for all years) such as, a lack of time for relaxation and the lack of holiday time account for mean DES scores of 3.42 and 2.84 respectively. Table 2 looked at the role of gender and marital status on mean DES scores. Female dental students reported higher stress levels (mean DES: female $=1.84$; males $=1.21, \mathrm{p}<0.05$ ) for family demands and lack of holiday time (mean DES: females $=3.25$; males $=2.51, \mathrm{p}<0.05)$ when compared to their male counterparts. Dental students who reported being married or common law, also reported higher stress levels than their single counterparts when discussing financial responsibilities, specifically pertaining to tuition costs (mean DES: 3.79 versus 3.06, p < 0.05) and size of current debt load (mean DES: 3.79 versus 2.97, $\mathrm{p}<0.05$ ). The role of gender was highlighted with female students reporting statistically higher stress levels pertaining to the social demands of family and the lack of holiday hours compared to their male counterparts (mean DES: 1.84 versus 1.21 and 3.25 versus 2.51 respectively). However, the overall lack of gender differences suggests that gender does not play a large role in student's stress levels. This trend can also be seen when looking at marital status. Slightly higher levels of stress were reported among students who were married or common law in terms of tuition costs (mean DES: 3.79 versus 3.06 ) and current debt load (mean DES: 3.79 versus 2.97).

Table 3 illustrates the role of current debt load on student stress levels, significantly higher levels of stress were reported among students incurring a current debt load of greater than $\$ 100,000$ dollars. This trend was also seen in those reporting a debt related interest payment of greater than $\$ 500$ dollars.

\section{Discussion}

This study presents current data on the role of student stress in dental education, and importantly presents data from western Canada, specifically the prairies. As the survey was conducted at the College of Dentistry, University of Saskatchewn the results cannot be generalized to all Canadian dental schools. High stress levels reported due to lack of time for relaxation among students at the University of Saskatchewan (means DES = 3.42) is consistent with Muirhead [10], who reported a mean DES 
Table 1 Mean dental environmental stress (DES) questionnaire scores and comparison among 4 years

\begin{tabular}{|c|c|c|c|c|c|c|c|}
\hline \multirow[t]{3}{*}{ Category } & \multirow[t]{3}{*}{ Stressor } & \multirow{3}{*}{$\begin{array}{l}\text { DES overall } \\
\text { Mean (SD) }\end{array}$} & \multicolumn{4}{|l|}{ DES by year } & \multirow[t]{3}{*}{ Sig. level } \\
\hline & & & Year 1 (2016) & Year 2 (2015) & Year 3 (2014) & Year 4 (2013) & \\
\hline & & & Mean (SD) & Mean (SD) & Mean (SD) & Mean (SD) & \\
\hline \multirow{10}{*}{$\begin{array}{l}\text { Social-related } \\
\text { stress }\end{array}$} & Living away from home & $0.92(1.14)$ & $1.14(0.96)$ & $1.13(1.25)$ & $1.00(1.38)$ & $0.35(0.61)$ & $0.039^{*}$ \\
\hline & $\begin{array}{l}\text { Accommodations not being conducive } \\
\text { for studying }\end{array}$ & $1.77(1.59)$ & $2.04(1.57)$ & $2.00(1.17)$ & $1.44(1.63)$ & $1.65(1.90)$ & 0.292 \\
\hline & Difficulty in making friends & $0.62(0.89)$ & $0.74(1.10)$ & $0.78(0.94)$ & $0.56(0.82)$ & $0.42(0.61)$ & 0.720 \\
\hline & Romantic relationships & $1.63(1.45)$ & $1.59(1.26)$ & $1.50(1.41)$ & $1.81(1.75)$ & $1.53(1.31)$ & 0.973 \\
\hline & Lack of time for relaxation & $3.42(1.33)$ & $3.24(1.13)$ & $3.55(1.43)$ & $3.81(1.39)$ & $2.95(1.27)$ & 0.109 \\
\hline & $\begin{array}{l}\text { Lack of holiday time compared to other } \\
\text { students }\end{array}$ & $2.84(1.39)$ & $2.96(1.10)$ & $2.90(1.55)$ & $2.63(1.64)$ & $2.89(1.24)$ & 0.893 \\
\hline & Social demands-family & $1.49(1.32)$ & $1.18(1.10)$ & $1.37(1.07)$ & $1.92(1.69)$ & $1.39(1.14)$ & 0.531 \\
\hline & Social demands-friends & $1.65(1.19)$ & $1.80(0.91)$ & $1.63(1.12)$ & $1.70(1.54)$ & $1.39(1.04)$ & 0.562 \\
\hline & Personal physical health & $1.97(1.62)$ & $1.60(1.61)$ & $1.60(1.70)$ & $2.62(1.79)$ & $1.95(1.08)$ & 0.088 \\
\hline & $\begin{array}{l}\text { Physical health of others-significant other/ } \\
\text { family }\end{array}$ & $1.51(1.40)$ & $1.18(1.05)$ & $1.47(1.59)$ & $2.14(1.58)$ & $1.13(1.15)$ & 0.145 \\
\hline \multirow{7}{*}{$\begin{array}{l}\text { Financial-related } \\
\text { stress }\end{array}$} & Financial responsibilities-living expenses & $2.33(1.50)$ & $2.32(1.49)$ & $2.05(1.87)$ & $2.52(1.45)$ & $2.37(1.21)$ & 0.757 \\
\hline & $\begin{array}{l}\text { Financial responsibilities-disposable } \\
\text { income }\end{array}$ & $2.18(1.58)$ & $2.38(1.47)$ & $1.71(1.72)$ & $2.39(1.67)$ & $2.11(1.49)$ & 0.499 \\
\hline & Financial responsibilities-tuition costs & $3.29(1.46)$ & $3.42(1.35)$ & $3.35(1.49)$ & $3.44(1.45)$ & $2.84(1.61)$ & 0.593 \\
\hline & Financial responsibilities-transportation & $1.62(1.28)$ & $1.35(1.03)$ & $1.33(1.14)$ & $1.88(1.37)$ & $1.84(1.50)$ & 0.404 \\
\hline & $\begin{array}{l}\text { Financial responsibilities-size of current } \\
\text { debt load }\end{array}$ & $3.23(1.60)$ & $3.00(1.62)$ & $3.21(1.62)$ & $3.42(1.58)$ & $3.26(1.69)$ & 0.793 \\
\hline & $\begin{array}{l}\text { Financial responsibilities-size of future } \\
\text { debt load }\end{array}$ & $3.36(1.59)$ & $3.42(1.47)$ & $3.28(1.60)$ & $3.38(1.67)$ & $3.32(1.73)$ & 0.979 \\
\hline & $\begin{array}{l}\text { Financial responsibilities-interest pay- } \\
\text { ments on debt }\end{array}$ & $2.81(1.61)$ & $2.50(1.44)$ & $2.83(1.65)$ & $3.12(1.61)$ & $2.74(1.82)$ & 0.531 \\
\hline \multirow[t]{11}{*}{$\begin{array}{l}\text { Clinical-related } \\
\text { stress }\end{array}$} & $\begin{array}{l}\text { Concern about manual dexterity and } \\
\text { clinical skills }\end{array}$ & $1.68(1.39)$ & $2.44(1.26)$ & $1.40(1.31)$ & $1.67(1.49)$ & $1.05(1.10)$ & $0.003^{*}$ \\
\hline & $\begin{array}{l}\text { Transition from pre-clinical to clinical } \\
\text { studies }\end{array}$ & $1.17(1.44)$ & $2.68(1.17)$ & $1.89(1.66)$ & $3.00(1.41)$ & $1.90(1.25)$ & $0.017^{*}$ \\
\hline & Completing clinical requirements & $2.84(1.48)$ & $2.93(1.33)$ & $2.90(1.33)$ & $3.19(1.62)$ & $2.21(1.44)$ & 0.149 \\
\hline & Clinical grading & $2.36(1.35)$ & $3.00(1.36)$ & $2.05(1.36)$ & $2.37(1.33)$ & $2.20(1.32)$ & 0.236 \\
\hline & $\begin{array}{l}\text { Differences in opinions of clinical faculty } \\
\text { and staff regarding clinical decision- } \\
\text { making and treatment }\end{array}$ & $2.88(1.39)$ & $3.05(1.60)$ & $2.75(1.29)$ & $3.19(1.36)$ & $2.42(1.26)$ & 0.237 \\
\hline & Supply of patients & $2.86(1.47)$ & $2.14(1.21)$ & $2.84(1.38)$ & $3.33(1.47)$ & $2.50(1.54)$ & 0.159 \\
\hline & Patient communication and management & $2.27(1.47)$ & $2.00(1.15)$ & $2.45(1.54)$ & $2.33(1.44)$ & $2.15(1.63)$ & 0.899 \\
\hline & $\begin{array}{l}\text { Confidence in own clinical decision- } \\
\text { making }\end{array}$ & $1.77(1.13)$ & $2.57(0.94)$ & $1.45(0.99)$ & $1.81(1.21)$ & $1.45(1.05)$ & $0.009^{*}$ \\
\hline & Adequacy of clinical supervision & $2.04(1.38)$ & $1.92(1.31)$ & $1.60(0.99)$ & $2.41(1.50)$ & $2.05(1.54)$ & 0.415 \\
\hline & $\begin{array}{l}\text { Patients attending scheduled appoint- } \\
\text { ments }\end{array}$ & $2.57(1.61)$ & $2.00(1.15)$ & $2.60(1.82)$ & $2.89(1.62)$ & $2.30(1.52)$ & 0.557 \\
\hline & Occupational/health hazards & $1.41(1.37)$ & $1.27(1.03)$ & $1.15(1.35)$ & $1.81(1.52)$ & $1.25(1.37)$ & 0.292 \\
\hline \multirow{7}{*}{$\begin{array}{l}\text { Academic- } \\
\text { related stress }\end{array}$} & Conducive teaching environment & $2.31(1.37)$ & $1.88(1.48)$ & $2.79(1.27)$ & $2.44(1.28)$ & $2.21(1.36)$ & 0.100 \\
\hline & Criticism of academic and/or clinical work & $2.78(1.38)$ & $3.04(1.46)$ & $3.15(1.35)$ & $2.74(1.40)$ & $2.00(1.00)$ & 0.064 \\
\hline & Approachability of faculty/staff & $2.16(1.45)$ & $1.76(1.45)$ & $2.85(1.39)$ & $2.15(1.35)$ & $2.00(1.53)$ & 0.068 \\
\hline & Communication with faculty/staff & $2.27(1.41)$ & $1.76(1.42)$ & $2.95(1.22)$ & $2.41(1.47)$ & $2.05(1.27)$ & $0.023^{*}$ \\
\hline & Rules/regulations of college & $2.62(1.61)$ & $2.32(1.75)$ & $2.85(1.56)$ & $2.89(1.60)$ & $2.37(1.50)$ & 0.481 \\
\hline & Expectation versus reality of dental school & $3.29(1.50)$ & $3.40(1.38)$ & $3.25(1.71)$ & $3.56(1.34)$ & $2.79(1.62)$ & 0.453 \\
\hline & Amount of course work & $3.28(1.30)$ & $3.40(0.82)$ & $3.20(1.24)$ & $3.59(1.39)$ & $2.80(1.64)$ & 0.306 \\
\hline
\end{tabular}


Table 1 continued

\begin{tabular}{|c|c|c|c|c|c|c|c|}
\hline \multirow[t]{3}{*}{ Category } & \multirow[t]{3}{*}{ Stressor } & \multirow{3}{*}{$\begin{array}{l}\text { DES overall } \\
\text { Mean (SD) }\end{array}$} & \multicolumn{4}{|l|}{ DES by year } & \multirow[t]{3}{*}{ Sig. level } \\
\hline & & & \multirow{2}{*}{$\begin{array}{l}\text { Year } 1 \text { (2016) } \\
\text { Mean (SD) }\end{array}$} & \multirow{2}{*}{$\begin{array}{l}\text { Year } 2 \text { (2015) } \\
\text { Mean (SD) }\end{array}$} & \multirow{2}{*}{$\begin{array}{l}\text { Year } 3 \text { (2014) } \\
\text { Mean (SD) }\end{array}$} & \multirow{2}{*}{$\begin{array}{l}\text { Year } 4 \text { (2013) } \\
\text { Mean (SD) }\end{array}$} & \\
\hline & & & & & & & \\
\hline & Difficulty of course work & $2.67(1.33)$ & $3.08(0.95)$ & $2.90(1.52)$ & $2.56(1.28)$ & $2.10(1.45)$ & 0.088 \\
\hline & Time available for learning & $3.32(1.31)$ & $3.48(1.05)$ & $3.55(1.28)$ & $3.56(1.28)$ & $2.55(1.47)$ & $0.051^{*}$ \\
\hline & $\begin{array}{l}\text { Fear of not being able to catch up if falling } \\
\text { behind in course work }\end{array}$ & $3.03(1.44)$ & $3.28(1.14)$ & $3.40(1.43)$ & $3.26(1.48)$ & $2.00(1.37)$ & $0.008^{*}$ \\
\hline & Fear of failing a course or year & $3.68(1.50)$ & $4.36(1.07)$ & $4.15(1.09)$ & $3.78(1.22)$ & $2.16(1.71)$ & $<0.001^{*}$ \\
\hline & Competition for grades & $1.96(1.58)$ & $2.36(1.75)$ & $2.11(1.52)$ & $1.93(1.41)$ & $1.35(1.56)$ & 0.170 \\
\hline & Uncertainty about future dental career & $2.01(1.48)$ & $2.40(1.58)$ & $2.05(1.61)$ & $1.74(1.16)$ & $1.84(1.61)$ & 0.469 \\
\hline & Examinations & $3.05(1.39)$ & $3.00(0.98)$ & $3.00(1.59)$ & $3.56(1.40)$ & $2.50(1.47)$ & 0.107 \\
\hline & $\begin{array}{l}\text { Lack of input into dental college decision- } \\
\text { making }\end{array}$ & $2.48(1.61)$ & $1.72(1.49)$ & $3.15(1.56)$ & $2.68(1.57)$ & $2.50(1.57)$ & $0.026^{*}$ \\
\hline & Clinical time allotted in curriculum & $2.24(1.32)$ & $2.41(1.28)$ & $2.40(1.09)$ & $2.41(1.55)$ & $1.70(1.17)$ & 0.176 \\
\hline & The language of teaching & $1.03(1.12)$ & $1.21(1.21)$ & $1.06(1.16)$ & $0.96(1.10)$ & $0.89(1.05)$ & 0.842 \\
\hline & $\begin{array}{l}\text { Knowledge transfer of information, meth- } \\
\text { ods and materials }\end{array}$ & $1.63(1.21)$ & $1.54(1.18)$ & $1.80(1.20)$ & $1.69(1.32)$ & $1.50(1.19)$ & 0.867 \\
\hline & The amount of material & $2.60(1.41)$ & $2.88(1.13)$ & $2.45(1.15)$ & $2.89(1.60)$ & $2.00(1.59)$ & 0.108 \\
\hline & The difficulty of material & $1.97(1.20)$ & $2.20(0.82)$ & $2.05(1.05)$ & $2.04(1.48)$ & $1.50(1.28)$ & 0.152 \\
\hline & Reference and information resources & $1.33(1.17)$ & $1.14(0.89)$ & $1.32(1.06)$ & $1.48(1.42)$ & $1.35(1.23)$ & 0.934 \\
\hline
\end{tabular}

* Statistically significant at $p<0.05$

of 3.14 for students at another Canadian dental school. Contextually, the University of Saskatchewan, begins classes in mid-August and continues until April or May (depending on year of program), the longer academic term coupled with the understanding that dental education places demands above and beyond the normal school hours, the findings are consistent with the literature [11]. Also consistent with the literature is the finding that clinical-related stressors, in particular those dealing with the transition from pre-clinical to clinical work, (i.e., clinical grading, patient supply and communication) are highest among 3rd year dental students (mean DES ranges from 2.33 to 3.33 ). Thus re-iterating the belief that the aforementioned transition marks an integral and stressful time in a student's dental education [12, 13]. High levels of stress were associated with differing opinions of clinical faculty and staff regarding decision making and treatment (mean DES scores ranged from 3.05 in 1st year to 2.42 in 4 th year). Clinical faculty is largely comprised of practicing or newly retired clinicians willing to provide their time, and thus bring differing experiences and backgrounds [14]. Currently, the College of Dentistry has no formalized program to calibrate clinical faculty, as some other dental institutions do. The high levels of stress attributed to this lack of clarity from faculty highlights the need for a calibration program to be implemented, potentially mitigating some stress levels among the students. Additionally, the incorporation of stress management strategies (i.e., mindfulness, therapy dogs) during high stress times (e.g., examination periods) should be explored by dental educators.

This study found that when looking at financial-related stressors, significant differences were seen between those with smaller debt loads $(\leq \$ 100,000)$ and those carrying larger debt loads $(\geq \$ 100,000)$, in all categories except tuition costs. Of interest is that higher stress due to living away from home was seen among those with lower debt loads (mean DES score of 1.34) compared to those with larger debts, while students living with their parents had significantly higher dental school entry debts. Similar findings were reported among Canadian dental students in Toronto, Canada [10]. This is important as the Financial Consumer Agency of Canada reported that tuition for the 2013-14 academic year rose 3.3 percent over the previous year and was expected to increase further from the reported Canadian average of $\$ 5772.00$ [15]. When 
Table 2 Mean dental environmental stress (DES) questionnaire scores and comparison among gender and marital status

\begin{tabular}{|c|c|c|c|c|c|c|c|}
\hline \multirow[t]{3}{*}{ Category } & \multirow[t]{3}{*}{ Stressor } & \multicolumn{2}{|c|}{ DES by gender } & \multirow[t]{3}{*}{ Sig. level } & \multicolumn{2}{|c|}{ DES by marital status } & \multirow[t]{3}{*}{ Sig. level } \\
\hline & & Male & Female & & Other & Married/common-law & \\
\hline & & Mean (SD) & Mean (SD) & & Mean (SD) & Mean (SD) & \\
\hline \multirow[t]{10}{*}{ Social-related stress } & Living away from home & $0.78(1.01)$ & $1.13(1.29)$ & 0.216 & $0.92(1.08)$ & $0.92(1.26)$ & 0.781 \\
\hline & $\begin{array}{l}\text { Accommodations not being condu- } \\
\text { cive for studying }\end{array}$ & $1.65(1.52)$ & $1.94(1.68)$ & 0.451 & $1.70(1.55)$ & $1.92(1.69)$ & 0.633 \\
\hline & Difficulty in making friends & $0.56(0.92)$ & $0.70(0.84)$ & 0.236 & $0.63(0.87)$ & $0.62(0.94)$ & 0.906 \\
\hline & Romantic relationships & $1.80(1.56)$ & $1.42(1.31)$ & 0.261 & $2.14(1.46)$ & $0.61(0.74)$ & $<0.001^{*}$ \\
\hline & Lack of time for relaxation & $3.33(1.31)$ & $3.53(1.36)$ & 0.471 & $3.35(1.34)$ & $3.55(1.30)$ & 0.533 \\
\hline & $\begin{array}{l}\text { Lack of holiday time compared to } \\
\text { other students }\end{array}$ & $2.51(1.42)$ & $3.25(1.26)$ & $0.015^{*}$ & $2.68(1.43)$ & $3.17(1.26)$ & 0.127 \\
\hline & Social demands-family & $1.21(1.16)$ & $1.84(1.44)$ & $0.036^{*}$ & $1.30(1.26)$ & $1.86(1.38)$ & 0.055 \\
\hline & Social demands-friends & $1.48(1.15)$ & $1.87(1.22)$ & 0.158 & $1.68(1.20)$ & $1.59(1.18)$ & 0.677 \\
\hline & Personal physical health & $1.74(1.35)$ & $2.25(1.89)$ & 0.311 & $2.11(1.61)$ & $1.66(1.63)$ & 0.160 \\
\hline & $\begin{array}{l}\text { Physical health of others-significant } \\
\text { other/family }\end{array}$ & $1.20(1.11)$ & $1.91(1.65)$ & 0.084 & $1.53(1.40)$ & $1.46(1.43)$ & 0.793 \\
\hline \multirow[t]{7}{*}{ Financial-related stress } & $\begin{array}{l}\text { Financial responsibilities-living } \\
\text { expenses }\end{array}$ & $2.20(1.41)$ & $2.50(1.61)$ & 0.449 & $2.12(1.47)$ & $2.79(1.47)$ & 0.055 \\
\hline & $\begin{array}{l}\text { Financial responsibilities-disposable } \\
\text { income }\end{array}$ & $2.10(1.56)$ & $2.29(1.62)$ & 0.652 & $2.02(1.58)$ & $2.52(1.55)$ & 0.165 \\
\hline & $\begin{array}{l}\text { Financial responsibilities-tuition } \\
\text { costs }\end{array}$ & $3.12(1.45)$ & $3.51(1.47)$ & 0.178 & $3.06(1.52)$ & $3.79(1.20)$ & $0.035^{*}$ \\
\hline & $\begin{array}{l}\text { Financial responsibilities-transpor- } \\
\text { tation }\end{array}$ & $1.54(1.27)$ & $1.71(1.29)$ & 0.535 & $1.53(1.18)$ & $1.81(1.47)$ & 0.510 \\
\hline & $\begin{array}{l}\text { Financial responsibilities-size of cur- } \\
\text { rent debt load }\end{array}$ & $3.15(1.58)$ & $3.33(1.64)$ & 0.515 & $2.97(1.64)$ & $3.79(1.40)$ & $0.025^{*}$ \\
\hline & $\begin{array}{l}\text { Financial responsibilities-size of } \\
\text { future debt load }\end{array}$ & $3.23(1.59)$ & $3.51(1.60)$ & 0.345 & $3.14(1.64)$ & $3.82(1.39)$ & 0.063 \\
\hline & $\begin{array}{l}\text { Financial responsibilities-interest } \\
\text { payments on debt }\end{array}$ & $2.70(1.56)$ & $2.95(1.68)$ & 0.511 & $2.58(1.60)$ & $3.29(1.56)$ & 0.068 \\
\hline \multirow[t]{11}{*}{ Clinical-related stress } & $\begin{array}{l}\text { Concern about manual dexterity } \\
\text { and clinical skills }\end{array}$ & $1.52(1.39)$ & $1.90(1.37)$ & 0.138 & $1.84(1.46)$ & $1.37(1.19)$ & 0.152 \\
\hline & $\begin{array}{l}\text { Transition from pre-clinical to clini- } \\
\text { cal studies }\end{array}$ & $2.20(1.37)$ & $2.76(1.50)$ & 0.072 & $2.37(1.44)$ & $2.57(1.48)$ & 0.475 \\
\hline & Completing clinical requirements & $2.53(1.53)$ & $3.22(1.33)$ & 0.059 & $2.75(1.44)$ & $3.04(1.56)$ & 0.386 \\
\hline & Clinical grading & $2.20(1.36)$ & $2.57(1.33)$ & 0.151 & $2.40(1.31)$ & $2.29(1.46)$ & 0.834 \\
\hline & $\begin{array}{l}\text { Differences in opinions of clinical } \\
\text { faculty and staff regarding clinical } \\
\text { decision-making and treatment }\end{array}$ & $2.71(1.43)$ & $3.11(1.33)$ & 0.222 & $2.88(1.28)$ & $2.89(1.62)$ & 0.877 \\
\hline & Supply of patients & $2.56(1.45)$ & $3.21(1.45)$ & 0.061 & $2.85(1.46)$ & $2.88(1.53)$ & 0.851 \\
\hline & $\begin{array}{l}\text { Patient communication and man- } \\
\text { agement }\end{array}$ & $2.17(1.39)$ & $2.40(1.56)$ & 0.521 & $2.20(1.38)$ & $2.41(1.62)$ & 0.681 \\
\hline & $\begin{array}{l}\text { Confidence in own clinical decision- } \\
\text { making }\end{array}$ & $1.73(1.19)$ & $1.81(1.06)$ & 0.608 & $1.77(1.12)$ & $1.75(1.17)$ & 0.807 \\
\hline & Adequacy of clinical supervision & $1.84(1.46)$ & $2.29(1.25)$ & 0.065 & $2.00(1.37)$ & $2.11(1.42)$ & 0.661 \\
\hline & $\begin{array}{l}\text { Patients attending scheduled } \\
\text { appointments }\end{array}$ & $2.15(1.46)$ & $3.06(1.67)$ & $0.018^{*}$ & $2.57(1.64)$ & $2.56(1.60)$ & 0.973 \\
\hline & Occupational/health hazards & $1.25(1.24)$ & $1.59(1.50)$ & 0.360 & $1.42(1.35)$ & $1.39(1.42)$ & 0.820 \\
\hline \multirow[t]{5}{*}{ Academic-related stress } & Conducive teaching environment & $2.12(1.29)$ & $2.56(1.45)$ & 0.169 & $2.13(1.35)$ & $2.69(1.36)$ & 0.098 \\
\hline & $\begin{array}{l}\text { Criticism of academic and/or clinical } \\
\text { work }\end{array}$ & $2.59(1.34)$ & $3.00(1.41)$ & 0.185 & $2.89(1.38)$ & $2.54(1.37)$ & 0.230 \\
\hline & Approachability of faculty/staff & $2.02(1.39)$ & $2.35(1.53)$ & 0.360 & $2.10(1.50)$ & $2.30(1.37)$ & 0.405 \\
\hline & Communication with faculty/staff & $2.04(1.37)$ & $2.55(1.43)$ & 0.087 & $2.17(1.49)$ & $2.47(1.25)$ & 0.273 \\
\hline & Rules/regulations of College & $2.69(1.59)$ & $2.53(1.65)$ & 0.616 & $2.70(1.59)$ & $2.43(1.65)$ & 0.447 \\
\hline
\end{tabular}


Table 2 continued

\begin{tabular}{|c|c|c|c|c|c|c|c|}
\hline \multirow[t]{3}{*}{ Category } & \multirow[t]{3}{*}{ Stressor } & \multicolumn{2}{|c|}{ DES by gender } & \multirow[t]{3}{*}{ Sig. level } & \multicolumn{2}{|c|}{ DES by marital status } & \multirow[t]{3}{*}{ Sig. level } \\
\hline & & Male & Female & & Other & Married/common-law & \\
\hline & & Mean (SD) & Mean (SD) & & Mean (SD) & Mean (SD) & \\
\hline & $\begin{array}{l}\text { Expectation versus reality of dental } \\
\text { school }\end{array}$ & $3.08(1.67)$ & $3.55(1.22)$ & 0.248 & $3.16(1.55)$ & $3.53(1.38)$ & 0.299 \\
\hline & Amount of course work & $3.06(1.38)$ & $3.58(1.15)$ & 0.060 & $3.26(1.27)$ & $3.33(1.40)$ & 0.592 \\
\hline & Difficulty of course work & $2.50(1.38)$ & $2.90(1.24)$ & 0.167 & $2.55(1.28)$ & $2.93(1.41)$ & 0.146 \\
\hline & Time available for learning & $3.19(1.44)$ & $3.48(1.11)$ & 0.412 & $3.29(1.28)$ & $3.37(1.38)$ & 0.716 \\
\hline & $\begin{array}{l}\text { Fear of not being able to catch up if } \\
\text { falling behind in course work }\end{array}$ & $2.80(1.47)$ & $3.33(1.37)$ & 0.100 & $3.00(1.39)$ & $3.10(1.56)$ & 0.659 \\
\hline & Fear of failing a course or year & $3.57(1.49)$ & $3.83(1.52)$ & 0.248 & $3.62(1.52)$ & $3.80(1.47)$ & 0.581 \\
\hline & Competition for grades & $2.00(1.53)$ & $1.90(1.67)$ & 0.650 & $2.06(1.50)$ & $1.72(1.75)$ & 0.206 \\
\hline & $\begin{array}{l}\text { Uncertainty about future dental } \\
\text { career }\end{array}$ & $1.86(1.61)$ & $2.21(1.28)$ & 0.173 & $1.87(1.41)$ & $2.31(1.61)$ & 0.240 \\
\hline & Examinations & $2.71(1.37)$ & $3.50(1.30)$ & $0.008^{*}$ & $2.95(1.34)$ & $3.28(1.51)$ & 0.247 \\
\hline & $\begin{array}{l}\text { Lack of input into dental college } \\
\text { decision-making }\end{array}$ & $2.22(1.50)$ & $2.82(1.70)$ & 0.084 & $2.33(1.50)$ & $2.77(1.79)$ & 0.237 \\
\hline & Clinical time allotted in curriculum & $2.26(1.36)$ & $2.21(1.30)$ & 0.821 & $2.18(1.25)$ & $2.36(1.47)$ & 0.701 \\
\hline & The language of teaching & $1.02(1.06)$ & $1.05(1.21)$ & 0.909 & $1.10(1.15)$ & $0.89(1.05)$ & 0.450 \\
\hline & $\begin{array}{l}\text { Knowledge transfer of information, } \\
\text { methods and materials }\end{array}$ & $1.63(1.23)$ & $1.64(1.20)$ & 0.943 & $1.67(1.31)$ & $1.55(0.98)$ & 0.947 \\
\hline & The amount of material & $2.38(1.46)$ & $2.88(1.32)$ & 0.108 & $2.68(1.40)$ & $2.43(1.45)$ & 0.397 \\
\hline & The difficulty of material & $1.83(1.11)$ & $2.15(1.29)$ & 0.288 & $1.95(1.21)$ & $2.00(1.20)$ & 0.819 \\
\hline & $\begin{array}{l}\text { Reference and information } \\
\text { resources }\end{array}$ & $1.28(1.16)$ & $1.39(1.20)$ & 0.619 & $1.32(1.20)$ & $1.36(1.13)$ & 0.729 \\
\hline
\end{tabular}

* Statistically significant at $p<0.05$

looking at the costs of dental education from 2010/11 to $2014 / 15$ average tuition costs rose from $\$ 15,062.00$ to $\$ 18,187.00$ per year [16]. It is not surprising then, that students with higher existing debt loads and interest payments had significantly higher self-reported stress levels relating to finances. Discussions around debt loads and costs of tuition are important, especially when post-secondary institutions are faced with increased operating costs and funding reductions.

\section{Conclusions}

The findings of this study are consistent with the literature, which states that financial and clinical workloads result in high stress levels among dental students. Dental educators must be cognizant of their responsibility to ensure that students, especially at the beginning of their education, have realistic expectations pertaining to issues such as workloads and costs of education. Of equal importance, all faculty especially clinical instructors, should be educated on grading systems and calibrated to help mitigate student stress as it pertains to faculty-student interactions. From a policy perspective, a formalized onboarding program for faculty and staff is recommended prior to interacting with students.

\section{Limitations}

This study was conducted at a single Canadian dental education institution (University of Saskatchewan) and the results cannot be generalized to other Canadian dental education institutions. However, the data was representative of the students enrolled at the College of Dentistry as evidenced by the high response rate. The findings represent the first time data on student stress was reported for a dental education institution located 
Table 3 Mean dental environmental stress (DES) questionnaire scores and comparison among debt load and debt interest payment

\begin{tabular}{|c|c|c|c|c|c|c|c|}
\hline \multirow[t]{3}{*}{ Category } & \multirow[t]{3}{*}{ Stressor } & \multicolumn{2}{|c|}{ DES by debt load } & \multirow[t]{3}{*}{ Sig. level } & \multicolumn{2}{|c|}{$\begin{array}{l}\text { DES by debt interest } \\
\text { payment }\end{array}$} & \multirow[t]{3}{*}{ Sig. level } \\
\hline & & $\leq \$ 100,000$ & $>\$ 100,000$ & & $\$ 0-500$ & $>\$ 500$ & \\
\hline & & Mean (SD) & Mean (SD) & & Mean (SD) & Mean (SD) & \\
\hline \multirow[t]{10}{*}{ Social-related stress } & Living away from home & $1.34(1.32)$ & $0.67(0.95)$ & $0.008^{*}$ & $0.90(1.14)$ & $1.00(1.19)$ & 0.722 \\
\hline & $\begin{array}{l}\text { Accommodations not being conducive for } \\
\text { studying }\end{array}$ & $1.77(1.55)$ & $1.81(1.62)$ & 0.973 & $1.81(1.60)$ & $1.71(1.54)$ & 0.880 \\
\hline & Difficulty in making friends & $0.65(0.85)$ & $0.60(0.93)$ & 0.654 & $0.64(0.94)$ & $0.53(0.64)$ & 0.947 \\
\hline & Romantic relationships & $1.82(1.53)$ & $1.51(1.42)$ & 0.310 & $1.68(1.45)$ & $1.43(1.55)$ & 0.460 \\
\hline & Lack of time for relaxation & $3.45(1.31)$ & $3.38(1.36)$ & 0.863 & $3.39(1.35)$ & $3.53(1.25)$ & 0.763 \\
\hline & $\begin{array}{l}\text { Lack of holiday time compared to other } \\
\text { students }\end{array}$ & $2.76(1.34)$ & $2.87(1.44)$ & 0.745 & $2.75(1.40)$ & $3.20(1.37)$ & 0.291 \\
\hline & Social demands-family & $1.17(1.01)$ & $1.73(1.48)$ & 0.119 & $1.32(1.14)$ & $2.33(1.80)$ & $0.043^{*}$ \\
\hline & Social demands-friends & $1.54(1.12)$ & $1.73(1.25)$ & 0.608 & $1.55(1.12)$ & $2.13(1.46)$ & 0.117 \\
\hline & Personal physical health & $1.92(1.67)$ & $2.02(1.62)$ & 0.728 & $1.84(1.61)$ & $2.67(1.59)$ & 0.058 \\
\hline & $\begin{array}{l}\text { Physical health of others-significant other/ } \\
\text { family }\end{array}$ & $1.19(1.26)$ & $1.83(1.47)$ & $0.047^{*}$ & $1.41(1.39)$ & $2.00(1.36)$ & 0.099 \\
\hline \multirow[t]{7}{*}{ Financial-related stress } & Financial responsibilities-living expenses & $1.68(1.32)$ & $2.77(1.48)$ & $0.001^{*}$ & $2.17(1.50)$ & $3.13(1.30)$ & $0.013^{*}$ \\
\hline & Financial responsibilities-disposable income & $1.68(1.53)$ & $2.55(1.54)$ & $0.014^{*}$ & $1.98(1.57)$ & $3.07(1.39)$ & $0.011^{*}$ \\
\hline & Financial responsibilities-tuition costs & $3.00(1.47)$ & $3.52(1.43)$ & 0.097 & $3.24(1.47)$ & $3.60(1.45)$ & 0.360 \\
\hline & Financial responsibilities-transportation & $1.51(1.28)$ & $1.73(1.27)$ & 0.363 & $1.47(1.15)$ & $2.40(1.55)$ & $0.024^{*}$ \\
\hline & $\begin{array}{l}\text { Financial responsibilities-size of current debt } \\
\text { load }\end{array}$ & $2.47(1.78)$ & $3.77(1.23)$ & $0.001^{*}$ & $3.07(1.62)$ & $4.13(1.12)$ & $0.015^{*}$ \\
\hline & $\begin{array}{l}\text { Financial responsibilities-size of future debt } \\
\text { load }\end{array}$ & $2.76(1.81)$ & $3.75(1.33)$ & $0.011^{*}$ & $3.20(1.63)$ & $4.13(1.25)$ & $0.030^{*}$ \\
\hline & $\begin{array}{l}\text { Financial responsibilities-interest payments } \\
\text { on debt }\end{array}$ & $2.18(1.59)$ & $3.25(1.49)$ & $0.004^{*}$ & $2.59(1.60)$ & $3.93(1.16)$ & $0.030^{*}$ \\
\hline \multirow[t]{11}{*}{ Clinical-related stress } & $\begin{array}{l}\text { Concern about manual dexterity and clinical } \\
\text { skills }\end{array}$ & $1.82(1.27)$ & $1.58(1.49)$ & 0.226 & $1.79(1.44)$ & $1.19(1.11)$ & 0.135 \\
\hline & Transition from pre-clinical to clinical studies & $2.49(1.50)$ & $2.42(1.42)$ & 0.880 & $2.52(1.41)$ & $2.13(1.59)$ & 0.227 \\
\hline & Completing clinical requirements & $2.68(1.25)$ & $2.98(1.60)$ & 0.294 & $2.89(1.38)$ & $2.75(1.84)$ & 0.811 \\
\hline & Clinical grading & $2.23(1.19)$ & $2.44(1.46)$ & 0.573 & $2.31(1.26)$ & $2.56(1.75)$ & 0.665 \\
\hline & $\begin{array}{l}\text { Differences in opinions of clinical faculty and } \\
\text { staff regarding clinical decision-making and } \\
\text { treatment }\end{array}$ & $2.82(1.45)$ & $2.94(1.38)$ & 0.800 & $2.91(1.40)$ & $2.81(1.42)$ & 0.859 \\
\hline & Supply of patients & $2.96(1.45)$ & $2.80(1.50)$ & 0.662 & $2.90(1.40)$ & $2.73(1.79)$ & 0.797 \\
\hline & Patient communication and management & $2.79(1.52)$ & $1.98(1.36)$ & $0.022^{*}$ & $2.34(1.48)$ & $2.00(1.41)$ & 0.401 \\
\hline & Confidence in own clinical decision-making & $2.00(1.34)$ & $1.64(0.98)$ & 0.286 & $1.82(1.16)$ & $1.60(1.06)$ & 0.757 \\
\hline & Adequacy of clinical supervision & $2.00(1.49)$ & $2.08(1.33)$ & 0.703 & $2.11(1.38)$ & $1.80(1.42)$ & 0.367 \\
\hline & Patients attending scheduled appointments & $2.68(1.72)$ & $2.50(1.56)$ & 0.609 & $2.61(1.64)$ & $2.40(1.55)$ & 0.661 \\
\hline & Occupational/health hazards & $1.57(1.52)$ & $1.32(1.28)$ & 0.616 & $1.50(1.41)$ & $1.06(1.18)$ & 0.246 \\
\hline \multirow[t]{9}{*}{ Academic-related stress } & Conducive teaching environment & $2.08(1.34)$ & $2.48(1.39)$ & 0.173 & $2.30(1.42)$ & $2.40(1.18)$ & 0.563 \\
\hline & Criticism of academic and/or clinical work & $2.92(1.40)$ & $2.66(1.38)$ & 0.399 & $2.84(1.41)$ & $2.38(1.19)$ & 0.267 \\
\hline & Approachability of faculty/staff & $1.87(1.38)$ & $2.37(1.49)$ & 0.148 & $2.12(1.47)$ & $2.31(1.45)$ & 0.604 \\
\hline & Communication with faculty/staff & $1.95(1.45)$ & $2.50(1.36)$ & 0.089 & $2.27(1.45)$ & $2.25(1.29)$ & 0.952 \\
\hline & Rules/regulations of College & $2.16(1.46)$ & $2.94(1.66)$ & $0.031^{*}$ & $2.58(1.58)$ & $2.75(1.84)$ & 0.796 \\
\hline & Expectation versus reality of dental school & $2.95(1.47)$ & $3.50(1.49)$ & 0.070 & $3.22(1.48)$ & $3.50(1.59)$ & 0.430 \\
\hline & Amount of course work & $3.34(1.12)$ & $3.25(1.44)$ & 0.964 & $3.32(1.23)$ & $3.13(1.67)$ & 0.855 \\
\hline & Difficulty of course work & $2.87(1.23)$ & $2.53(1.39)$ & 0.276 & $2.68(1.29)$ & $2.63(1.59)$ & 0.957 \\
\hline & Time available for learning & $3.53(1.25)$ & $3.17(1.35)$ & 0.219 & $3.43(1.25)$ & $2.81(1.51)$ & 0.136 \\
\hline
\end{tabular}


Table 3 continued

\begin{tabular}{|c|c|c|c|c|c|c|c|}
\hline \multirow[t]{3}{*}{ Category } & \multirow[t]{3}{*}{ Stressor } & \multicolumn{2}{|c|}{ DES by debt load } & \multirow[t]{3}{*}{ Sig. level } & \multicolumn{2}{|c|}{$\begin{array}{l}\text { DES by debt interest } \\
\text { payment }\end{array}$} & \multirow[t]{3}{*}{ Sig. level } \\
\hline & & $\leq \$ 100,000$ & $>\$ 100,000$ & & & & \\
\hline & & Mean (SD) & Mean (SD) & & Mean (SD) & Mean (SD) & \\
\hline & $\begin{array}{l}\text { Fear of not being able to catch up if falling } \\
\text { behind in course work }\end{array}$ & $3.16(1.40)$ & $2.94(1.49)$ & 0.536 & $3.11(1.38)$ & $2.69(1.74)$ & 0.421 \\
\hline & Fear of failing a course or year & $3.79(1.28)$ & $3.58(1.65)$ & 0.898 & $3.74(1.45)$ & $3.31(1.70)$ & 0.285 \\
\hline & Competition for grades & $2.00(1.49)$ & $1.94(1.67)$ & 0.786 & $1.95(1.58)$ & $2.06(1.69)$ & 0.809 \\
\hline & Uncertainty about future dental career & $1.76(1.38)$ & $2.14(1.49)$ & 0.250 & $1.92(1.41)$ & $2.25(1.65)$ & 0.475 \\
\hline & Examinations & $2.95(1.35)$ & $3.12(1.44)$ & 0.494 & $3.11(1.35)$ & $2.75(1.61)$ & 0.453 \\
\hline & $\begin{array}{l}\text { Lack of input into dental college decision- } \\
\text { making }\end{array}$ & $2.00(1.45)$ & $2.85(1.64)$ & $0.016^{*}$ & $2.51(1.63)$ & $2.44(1.55)$ & 0.957 \\
\hline & Clinical time allotted in curriculum & $2.36(1.19)$ & $2.18(1.41)$ & 0.448 & $2.18(1.28)$ & $2.60(1.50)$ & 0.302 \\
\hline & The language of teaching & $1.00(1.09)$ & $1.08(1.15)$ & 0.761 & $0.97(1.14)$ & $1.38(1.02)$ & 0.097 \\
\hline & $\begin{array}{l}\text { Knowledge transfer of information, methods } \\
\text { and materials }\end{array}$ & $1.59(1.17)$ & $1.67(1.26)$ & 0.850 & $1.60(1.19)$ & $1.81(1.38)$ & 0.616 \\
\hline & The amount of material & $2.82(1.25)$ & $2.18(1.09)$ & 0.161 & $2.64(1.36)$ & $2.44(1.71)$ & 0.479 \\
\hline & The difficulty of material & $2.18(1.09)$ & $1.81(1.27)$ & 0.071 & $1.93(1.13)$ & $2.13(1.54)$ & 0.842 \\
\hline & Reference and information resources & $1.56(1.34)$ & $1.20(1.02)$ & 0.247 & $1.32(1.19)$ & $1.44(1.09)$ & 0.563 \\
\hline
\end{tabular}

* Statistically significant at $p<0.05$

in western Canada thus providing insight for educators into student stress levels. Further research is needed to determine if similar results would be reported by dental students across Canada.

\section{Additional files}

Additional file 1. Dental Environment Stress Survey.

Additional file 2. Reliability of stress factors (subscales) of DES

Questionnaire.

Additional file 3. Socio-demographic characteristics of the participants.

\section{Abbreviations}

DES: dental environment survey; CMHA: Canadian Mental Health Association; GPA: grade point average; DMD: Doctor of Medicine in Dentistry; PSS-10: Perceived Stress Scale 10; SPSS: statistical Package for the Social Sciences.

\section{Authors' contributions}

AH contributed to the secondary literature search, the writing and editing of the manuscript. JH contributed to the initial literature search, the initial drafts of the 'introduction' and 'methods' sections of the manuscript and supervised the collection of data. CK provided assistance with data analysis and interpretation. GU contributed to the study conception and data collection. All authors read and approved the final manuscript.

\section{Author details}

${ }^{1}$ College of Dentistry, University of Saskatchewan, 105 Wiggins Rd, Saskatoon, SK S7N 5E4, Canada. ${ }^{2}$ Canadian Centre for Health and Safety in Agriculture-CCHSA, University of Saskatchewan, Saskatoon, SK S7N 2Z4, Canada.

\section{Acknowledgements}

The authors wish to thank Simon-Hannes Haimanot, Saud Ahmad, Adam Abdo, and all participants who were students at the College of Dentistry, University of Saskatchewan at the time of study.

\section{Competing interests}

The authors declare that they have no competing interests.

\section{Availability of data and materials}

The data generated and analyzed in this study are not publicly available as further analysis is ongoing. Data are available from the corresponding author on reasonable request.

\section{Consent for publication}

Not applicable.

\section{Ethics approval and consent to participate}

Ethics approval was obtained from the University of Saskatchewan, Behavioral Research Ethics Board (Beh-REB), Reference Number \# 12-122. All participants provided their free and written, informed consent.

\section{Funding}

Minimal funding was provided by the College of Dentistry, as the survey pertained to a student led research initiative.

\section{Publisher's Note}

Springer Nature remains neutral with regard to jurisdictional claims in published maps and institutional affiliations.

Received: 22 March 2017 Accepted: 22 November 2017

Published online: 08 December 2017 


\section{References}

1. Canadian Mental Health Association. Stress. 2016. http://www.cmha.ca/ mental_health/stress/\#.VOiCjeSm9Tc. Accessed 27 May 2016.

2. Sanders $A E$, Lushington $K$. Effect of perceived stress on student performance in dental school. J Dent Educ. 2002;66(1):75-81.

3. Humphris $\mathrm{G}$, et al. Psychological stress in undergraduate dental students: baseline results from seven European dental schools. Eur J Dent Educ. 2002;6:22-9.

4. Alzahem AH, van der Molen HT, Alaujan AH, Schmidt HG, Zamakhshary $\mathrm{MH}$. Stress amongst dental students: a systematic review. Eur J Dent Educ. 2011;15(1):8-18.

5. Pau A, et al. Emotional intelligence and perceived stress in dental undergraduates: a multinational survey. J Dent Educ. 2007;71:197-204.

6. Polychronopoulou A, Divaris K. Dental students' perceived sources of stress: a multi-country study. J Dent Educ. 2009;73:631-9.

7. Al-Saleh $\mathrm{S}$, et al. Survey of perceived stress-inducing problems among dental students, Saudi Arabia. Saudi Dent J. 2010;22:83-8.

8. Elani HW, et al. A systematic review of stress in dental students. J Dent Educ. 2014;78(2):226-42.
9. McDonald JH. Handbook of biological statistics. 3rd ed. Baltimore: Sparky House Publishing; 2014

10. Muirhead V, Locker D. Canadian dental students' perceptions of stress. J Can Dent Assoc. 2007;73(4):323.

11. Garabee WH, Zucker SB, Selby GR. Perceived sources of stress among dental students. J Am Dent Assoc. 1980;100(6):853-7.

12. Murphy RJ, Gray SA, Sterling G, Reeves K, DuCette J. A comparative study of professional student stress. J Dent Educ. 2009;73(3):328-37.

13. Grandy TG, Westerman $\mathrm{GH}$, Combs $\mathrm{CE}$, Turner $\mathrm{CH}$. Perceptions of stress among third year dental students. J Dent Educ. 1989;53(12):718-21.

14. Chapnick L, Chapnick A. The part-time clinical instructor in the undergraduate dental clinic. J Can Dent Assoc. 1998;64(5):374-5.

15. Financial Consumer Agency of Canada. Budget for student life-how much will your post-secondary education cost? 2014. http://www. fcac-acfc.gc.ca/Eng/forConsumers/lifeEvents/payingPostSecEd/Pages/ Budgetfo-Unbudget.aspx. Accessed 5 Sept 2016.

16. Statistics Canada. Undergraduate tuition fees. http://www.statcan.gc.ca/ tables-tableaux/sum-som/l01/cst01/educ50a-eng.htm. Accessed 5 Sept 2016

\section{Submit your next manuscript to BioMed Central and we will help you at every step:}

- We accept pre-submission inquiries

- Our selector tool helps you to find the most relevant journal

- We provide round the clock customer support

- Convenient online submission

- Thorough peer review

- Inclusion in PubMed and all major indexing services

- Maximum visibility for your research

Submit your manuscript at www.biomedcentral com/submit 